\section{Lesões por esforços repetitivos/ distúrbios osteomusculares relacionados ao trabalho em cirurgiões-dentistas}

\section{Work related musculoskeletal disorders in dentists}

\author{
Gilsée Ivan Regis Filho' \\ Glaycon Michels ${ }^{2}$ \\ Ingeborg Sell ${ }^{3}$ \\ 1 Departamento de Estomatologia da Universidade Federal de Santa Catarina \\ ${ }^{2}$ Curso de Pós-Graduação em Odontologia da Universidade do Sul de Santa \\ Catarina \\ ${ }^{3}$ Universidade Regional de Blumenau
}

Correspondência: Gilsée Ivan Regis Filho, Dr. Departamento de Estomatologia - Centro de Ciências da Saúde - Universidade Federal de Santa Catarina. Campus Universitário - Trindade - CEP 88040-900 Florianópolis - Santa Catarina.e-mail: gregis@brturbo.com.br

\section{Resumo}

As patologias denominadas genericamente de Lesões por Esforços Repetitivos (LERs)/Distúrbios Osteomusculares Relacionados ao Trabalho (DORTs), apresentando sinais e sintomas de inflamações dos músculos, tendões, fáscias e nervos dos membros superiores, cintura escapular e pescoço, entre outros, têm chamado a atenção não só pelo aumento da sua incidência mas por existirem evidências de sua associação com o ritmo de trabalho. $\mathrm{O}$ exercício profissional obriga que cirurgiões-dentistas utilizem na execução das tarefas os membros superiores e estruturas adjacentes, freqüentemente com repetitividade de um mesmo padrão de movimento, compressão mecânica das estruturas localizadas na região, assumindo posturas incorretas, utilizando força excessiva e ainda, na maioria dos casos, trabalhando sob pressão temporal. O presente estudo utilizou o método epidemiológico transversal para buscar evidências da existência da relação entre as tarefas executadas pelo cirurgião-dentista e as LERs/DORTs. Os estudos empíricos revelaram presença de associação estatisticamente significante entre os dois sexos e as patologias. O sexo feminino apresenta mais lesões que o masculino. Ombro/braço $(39,40 \%)$, punho/ mão $(18,30 \%)$ e pescoço $(17,20 \%)$ são as regiões mais afetadas. Finalmente, o presente estudo é mais um indicativo de que as tarefas executadas estão expondo esses profissionais a um risco considerável de adquirir LERs/DORTs.

Palavras-chave: Lesões por esforços repetitivos. Desordens por trauma cumulativo. DORT. 


\section{Abstract}

Conditions generally named Cumulative Trauma Disorders (CTD) / Work Related Musculoskeletal Disorders (WRMD), that present with signs and symptoms of inflammation in the muscles, tendons, fascia and nerves of the upper limb, shoulder girdle and neck, among others, have been observed not only on account of their incidence but also for their association with work. Dentists are forced to use upper and adjacent structures during their professional activities and frequently perform the same movement pattern, or cause mechanical compression of structures in that area, because of adopting incorrect postures and excessive force, and in most cases due time pressure. This study has employed a cross-sectional epidemiological method to look for evidence on the relation between the tasks dentists perform and CTD/WRMD. Empirical studies have shown the presence of a statistically significant association between both genders and the conditions. Women have presented a greater number of lesions compared to men. Shoulder/arm $(39.40 \%)$, wrist/hand (18.30\%), and neck (17.20\%) are the most frequently affected regions. In the present study, analysis of cinematic data has revealed that the main activities dentists perform force two or more regions of the body to positions considered of medium and high risk. Analysis of electromyography signs showed great compromise of flexor and extensor muscle groups of carpi and trapezius. Finally, the present study is one more indication that the tasks carried out are exposing professionals to a considerable risk of acquiring CTD/WRMD.

Keywords: Repetitive strain injury. Cumulative trauma disorders. WRMD.

\section{Introdução}

Na Inglaterra do século XVIII, durante a Revolução Industrial, e com a conseqüente passagem do sistema artesanal ou familiar para o sistema fabril de produção, as ainda incipientes empresas começaram a se preocupar com custos, métodos de fabricação e planejamento.

Um pouco mais tarde, no século XIX, a fábrica chega ao resto da Europa e aos Estados Unidos da América e, graças ao transporte dinamizado pelas ferrovias, um grande contingente de indivíduos passou a viver nas cidades e a trabalhar na indústria.

No rigor das primeiras fábricas, as jornadas de trabalho costumavam ser de quinze horas, seis dias por semana, empregando mulheres e crianças, sendo o trabalho estafante, monótono, com severa disciplina e acidentes freqüentes ${ }^{1}$.

No início do século XX, com Frederick Winslow Taylor em 1911 e Henri Fayol em 1916, as revolucionárias linhas de produção tiveram seu foco de atenção voltado para a racionalização de métodos, tempos e movimentos ${ }^{2,3}$.

A partir de 1945, com Joseph Moses Juran e Willians Edwards Deming, e da segunda metade do século XX, com Peter Ferdinand Drucker, Kaoru Ishikawa, Douglas McGregor e Amitai Etzioni, chegou-se à qualidade total, just in time, série ISO, motivação, desenvolvimento gerencial, tornando definitivamente o trabalho moderno baseado em padrões de produtivida$\mathrm{de}^{4-9}$.

Entretanto, com o aumento significativo da industrialização e da produtividade cresceram, também, de maneira bastante evidente, os relatos de LERs/DORTs, chegando-se a considerar a existência de uma nova epidemia industrial ${ }^{10}$.

As patologias denominadas genericamente de LERs/DORTs, apresentando sinais e sintomas de inflamações dos músculos, tendões, fáscias e nervos dos membros superiores, cintura escapular e pescoço, entre outros, têm chamado a atenção não só pelo aumento de sua incidên- 
cia mas por existirem evidências de sua associação com o ritmo de trabalho. Essas patologias, em geral, não têm um tratamento difícil, mas sim possuem uma má evolução, causando dor, perda de força e edema, sendo responsáveis por uma parcela significativa das causas da queda da performance no trabalho.

Normalmente se encontra entre as origens de LERs/DORTs as atividades no trabalho que exijam força excessiva com as mãos, posturas inadequadas dos membros superiores, repetitividade de um mesmo padrão de movimento, compressão mecânica das estruturas dos membros superiores e regiões anexas e o tempo insuficiente para a realização de um determinado trabalho ${ }^{11}$.

Por outro lado, causas do aparecimento de LERs/DORTs também são encontradas nas atividades domésticas de maior exigência com as mãos, como jardinagem, tricotar, costurar e fazer crochê, no uso de instrumentos musicais, como por exemplo piano, e em atividades esportivas que exijam grande esforço dos membros superiores, a exemplo do vôlei, tênis e golfe ${ }^{12}$.

Usuários de terminais de vídeo constituem também, de maneira geral, um grupo de risco ${ }^{13}$. Estudos realizados sobre as condições de trabalho de operadores desses terminais demonstraram que a freqüência de queixas quanto aos problemas musculares e visuais eram menores onde a organização do trabalho permitia uma certa autonomia em relação à execução da tarefa e onde havia pausas fixas intercaladas por tarefas de natureza diferente; entretanto fatores físicos e ambientais estariam por trás do aparecimento desses sintomas ${ }^{14}$.

Recentemente se observou um aumento da incidência de LERs/DORTs entre intérpretes de linguagem de sinais, devido a uma interação entre exigências do trabalho e estilo de trabalho, com mais estresse biomecânico sobre as extremidades superiores. Aqueles com sintomatologia dolorosa faziam geralmente menos pausas recreativas, apresentavam desvios mão/punho fugindo da posição neutra, moviam mãos/dedos mais rapidamente e possuíam maior tendência para relatar dor ${ }^{15}$.

Matte, Baker e Honchar, e Erdil, Dickerson e Glackin alegam que tais ocorrências aumentaram graças a melhores critérios de diagnósticos e definições dadas pelo Nacional Institute for Occupational Safety and Healty (NIOSH) para as LERs/ DORTs ligadas ao trabalho ${ }^{16,17}$.

Entretanto, Hadler tem opinião diversa. Para este autor, o aumento dos relatos de LERs/DORTs naquele país vem de maiores exigências do trabalho, com dor e fadiga muscular, ou de interesses financeiros, como defeitos nos incentivos do sistema de compensação trabalhista e a cumplicidade ou a ingenuidade dos médicos ${ }^{18}$.

Embora milhões de americanos sofram de LERs/DORTs ou trabalhem em empregos onde é grande este risco, existem ainda controvérsias quanto à sua verdadeira incidência, empregos ou tarefas de risco, tratamentos, intervenções ergonômicas e estratégias de prevenção ${ }^{19}$.

No Brasil, a partir de 1985, também houve um significativo aumento dos casos de LERs/DORTs de 4,69\% das doenças profissionais para $41,77 \%$ em 1988. As mulheres são as mais facilmente afetadas, talvez, pela jornada doméstica, menor número de fibras musculares, menor capacidade de armazenar e converter glicogênio em energia útil e por serem entregues a elas, na indústria, as atividades repetitivas que exigem maior habilidade. $O$ quadro se agrava, principalmente, durante a gestação e a menopausa $^{20,21}$.

Para Couto, se algum trabalhador tiver alguma doença do trabalho ou ocupacional que o deixe incapacitado, ele tem mais de $1 / 3$ de chance de essa doença ser decorrente de LERs/DORTs. Para tanto, basta que exista a conjugação de fatores de risco relacionados ao trabalho como: biomecânicos, psicossociais, administrativos e outros fatores contributivos, para que possa se esperar predisposição à ocorrência dessas patologias ${ }^{22}$.

As LERs/DORTs como doença ocupacional devem ser analisadas dentro do 
trinômio homem, máquina e ambiente de trabalho. Fatores econômico-financeiros e de produtividade obrigam, por exemplo, os digitadores a um excesso de toques por hora entre 10 e 15.000 quando os tendões não toleram mais que 2.000 e de aumento da jornada de trabalho com a utilização do duplo emprego, possibilitando que $20 \%$ dos digitadores venham a desenvolver algum tipo de LERs/DORTs ${ }^{15}$. Pessoas que executam tarefas altamente repetitivas e forçadas têm 29 vezes mais risco de contrair essas patologias em punhos e mãos ${ }^{23-26}$.

$\mathrm{Na}$ avaliação de alguns estudos ${ }^{27,28}$, as principais ocupações associadas às LERs/ DORTs são:

- Trabalhadores de montagem de aviões, de carros e do setor de eletrônica;

- Engarrafadores, empacotadores e transportadores;

- Trabalhadores da produção de carvão (coque), da construção civil, da produção de pneus e artefatos de borracha;

- Polidores, açougueiros, caixas, motoristas, inspetores, mineiros, músicos, secretárias, datilógrafos e garçonetes;

- Cortadores e costureiras de tecidos, preparadores de alimentos, fabricantes de malas e processadores de carnes e aves;

- Verificadores de gêneros alimentícios e fabricantes de produtos de tecelagem;

- Fabricantes de metais, estofadores e usuários de ferramentas vibratórias;

- Trabalhadores de jornais e da manufatura de artigos oftalmológicos; e

- Trabalhadores dos correios, intérpretes de linguagem de sinais e usuários de terminais de vídeo.

Parece claro o caráter diversificado da epidemiologia da doença quanto aos grupos profissionais atingidos, porém os cirurgiões-dentistas praticamente não são citados na literatura científica. Tal fato ocorre em virtude de que as pesquisas e os dados epidemiológicos disponíveis, associando a patologia aos referidos profissionais, são escassos. Somente alguns pou- cos autores, nas últimas três décadas, têm relacionado os procedimentos odontológicos com o aparecimento de LERs / DORTs em cirurgiões-dentistas e higienistas dentais. Entre esses autores se encontram Eccles e Powell, que analisaram 231 cirurgiões-dentistas no País de Gales e constataram que $5 \%$ deles trabalhavam de pé e que a maioria se referia a fadiga apreciável. Do mesmo modo, a posição de empunhar a ferramenta peça de mão tem grande importância ergonômica, já que o posicionamento incorreto leva à tensão muscular ${ }^{29}$.

Por outro lado, para Lawrence, é bastante comum entre cirurgiões-dentistas a degeneração dos discos intervertebrais da região cervical. Outras patologias, como a periartrite escápulo-umeral ou bursite, a hipertrofia muscular observada no membro mais utilizado e a contratura muscular fisiológica, são comuns nesta categoria profissional $^{30}$. Encontra-se, também, desigualdade na altura do ombro em vários cirurgiões-dentistas, inflamação das bainhas tendinosas e artrite das mãos ${ }^{31,32}$.

Estudo realizado por Regis Filho e Lopes encontrou, entre profissionais cirurgiões-dentistas participantes do II Encontro de Ex-alunos do Curso de Graduação em Odontologia da Universidade Federal de Santa Catarina - 1996 (SC), 25,81\% de homens e $52,94 \%$ de mulheres com sintomatologia de LERs/DORTs, sendo que ombros e punhos, ambos com $44,44 \%$ cada um, eram os locais onde os homens relatavam maior sintomatologia dolorosa. De acordo com os autores, $31,57 \%, 36,84 \%$ e $26,32 \%$ das mulheres apresentavam dor no punho, ombro e dedos, respectivamente ${ }^{11}$.

Números semelhantes são encontrados no trabalho de Santos Filho, onde 58\% dos cirurgiões-dentistas do Serviço Público de Saúde de Belo Horizonte (MG) relataram dor global, sendo que 22\%, 21\%, 20\% e $17 \%$ apresentavam dor no membro superior, coluna, pescoço e ombro, respectivamente $^{33}$.

Bauer apresenta um estudo de caso de Síndrome do Túnel do Carpo em um higi- 
enista dental, concluindo que o reconhecimento da classe médica em associar a patologia a uma doença de caráter ocupacional está se tornando uma prática um pouco mais comum. No entanto, para Holtzhausen, são necessárias mais pesquisas antes de se propor medidas preventi$\operatorname{vas}^{34,35}$.

Outro autor, Schlim, citado por Lopes e Villanacci Neto, também afirma que há uma grande incidência da Síndrome do Túnel do Carpo entre cirurgiões-dentistas, principalmente periodontistas, endodontistas, naqueles que praticam exodontias em grande número e em higienistas dentais $^{36}$.

Alguns autores, como Cândido, Bittencourt, Regis Filho; Martins, Fadel e Regis Filho; e Fadel, Pietrobon, Patiño e Regis Filho, apresentam relatos de caso de LERs/DORTs, respectivamente, de Cisto Sinovial de Punho, Síndrome do Túnel Carpal e Dedo em Gatilho em cirurgiãodentista, sendo todos do sexo feminino, onde a prática odontológica contribuiu, decididamente, para o desencadeamento da LER/DORT e que trouxeram forte limitação funcional nos dois últimos $\operatorname{casos}^{37-39}$.

Por outro lado, para Barnhart, Demers, Miller, Longstreth e Rosenstock, os instrumentos vibratórios e movimentos contínuos contribuem para o surgimento da Síndrome do Túnel do Carpo em cirurgiões-dentistas e higienistas dentais, o que está de acordo com o que afirma Jetzer, para quem o uso desses instrumentos, como os utilizados no desgaste de rebarbas de acrílico no acabamento de próteses totais, desempenharia importante papel no desencadeamento dessa síndrome $\mathrm{e}^{40,41}$.

Regis Filho, Fadel, Pietrobon, Zmijvski e Klug analisaram a vibração produzida pelos instrumentos rotatórios de alta e baixa-rotação utilizados na prática odontológica e concluíram que, a princípio, seriam necessários 17 anos e uma rotina de trabalho de 6 horas diárias para que até $10 \%$ dos cirurgiões-dentistas desenvolvessem alguma patologia associada à vibração transmitida para a mão/braço como a
Síndrome do Túnel Carpal e a Síndrome da Vibração Mão/Braço ${ }^{42}$.

Para o cirurgião-dentista, a repetição de gestos e a manutenção de contra-resistências, bem como a postura dos membros superiores, com atividades que exijam constante flexão e extensão do punho, a compressão mecânica das bases das mãos com instrumentos periodontais e endodônticos curtos e inadequados e que necessitam da utilização de força na base da mão, são as causas ocupacionais imediatas da Síndrome do Túnel do Carpo ${ }^{34,41}$.

Outro fator a ressaltar, segundo Pece, é que são comuns os casos de inadequação operador/equipamento/instrumento nas áreas biológicas, obrigando o profissional a assumir posturas incorretas de trabalho e, conseqüentemente, na execução da tarefa ocorrem microtraumatismos, ou seja, a destruição de pequeno número de células, cuja somatória pode originar as tecnopatias odontológicas e entre elas as LERs/ DORTs $^{43}$.

O exercício profissional obriga que cirurgiões-dentistas utilizem na execução das tarefas os membros superiores e estruturas adjacentes, sendo as mãos particularmente mais exigidas, freqüentemente com repetitividade de um mesmo padrão de movimento em virtude da atividade clínica, compressão mecânica das estruturas localizadas na região em função de instrumentos inadequados, assumindo posturas incorretas por necessidade de técnicas operatórias e utilizando força excessiva em virtude das características próprias de algumas patologias, e ainda, na maioria dos casos, trabalhando sob pressão temporal. Assim, as LERs/DORTs representam a conseqüência tardia do mau uso crônico de um delicado conjunto mecânico que são os membros superiores e regiões adjacentes, seja pelo uso da força excessiva, por compressão mecânica, posturas desfavoráveis das articulações ou alta repetitividade, salientando que, como fator isolado, o esforço excessivo se mostra mais importante que os efeitos deletérios de cada um. 
Observa-se pela literatura pesquisada evidências de que um número significativo de cirurgiões-dentistas são ou foram portadores de LERs/DORTs perfeitamente já diagnosticadas. Entretanto, esses relatos ou eram oriundos de amostras pequenas e não representativas, ou provinham de estudos de casos com sub-grupos, originando controvérsias e não se permitindo afirmar, ainda, que o cirurgiãodentista pertence a um grupo profissional que reúne as características necessárias para que possa ser incluído nos grupos preferenciais de risco de adquirir algum tipo de LER/DORT.

No presente estudo, procurar-se-á detectar se o cirurgião-dentista pertence a um grupo profissional exposto a risco considerável de adquirir algum tipo de LER/ DORT, desde que certas condições inerentes às tarefas profissionais, aí consideradas força excessiva, posturas incorretas, alta repetitividade de um mesmo padrão de movimento e compressão mecânica dos tecidos, aliadas às características individuais, estejam presentes. Será realizado estudo epidemiológico transversal retrospectivo.

Por opção metodológica se aborda sómente aspectos epidemiológicos, excluindo-se do presente trabalho outros fatores, como uma análise mais profunda no campo psicossocial, aqueles decorrentes das interações com instrumentos, os individuais biológicos e/ou genéticos, a exposição a agentes externos químicos e/ou biofísicos, os legais e os de organização temporal do trabalho, relacionados com as LERs/DORTs em cirurgiões-dentistas, o que determina os limites do presente estudo.

\section{Material e métodos}

O presente trabalho utilizou um estudo epidemiológico transversal para buscar evidências da existência da relação entre as tarefas executadas pelo cirurgião-dentista e as LERs/DORTs.

Utilizou-se para coleta de dados epidemiológicos um protocolo denominado "In- quérito Sócio/Sanitário" e que foi adaptado de Lech e Hoefel, objetivando pesquisar as manifestações sentidas ou não, passadas ou presentes, relacionadas às LERs/ DORTs, provocadas pelo trabalho do cirurgião-dentista, bem como o perfil socioeconômico destes profissionais ${ }^{44}$.

O protocolo do presente estudo está dividido em três partes: uma destinada a identificação do pesquisado quanto a aspectos sociais e pessoais; outra, relacionada ao perfil profissional; e uma terceira abordando propriamente as manifestações fisiopatológicas das LERs/DORTs. O protocolo foi pré-testado em um estudo piloto.

O protocolo "Inquérito Sócio/Sanitário" foi enviado pelo sistema de carta e cartão-resposta tipo convencional, ou seja, o conteúdo foi inserido em envelopes específicos, previamente personalizados, para os cirurgiões-dentistas cadastrados no Conselho Regional de Odontologia de Santa Catarina no ano 2.000, totalizando 3.618 questionários. Destes, 523 foram devolvidos por mudança de endereço, 771 retornaram devidamente preenchidos, e dos restantes 2.324 não se obteve resposta. O número de questionários devolvidos preenchidos (771) corresponde a 24,91\% dos válidos. De acordo com a Empresa Brasileira de Correios e Telégrafos, baseada na experiência adquirida em décadas oferecendo essa modalidade de serviço, nesse tipo de pesquisa se espera um retorno da ordem de não mais que $10 \%$. Embora esse argumento não seja científico, é o único disponível na literatura. Assim, apenas comparativamente, o número de questionários que retornaram pode ser considerado um percentual excelente ${ }^{45}$.

A metodologia estatística utilizada para tratamento dos dados epidemiológicos consistiu em: para o agrupamento dos casos com presença de LER/DORT foram utilizadas a Análise Fatorial de Correspondência Múltipla (AFCM), a Classificação Hierárquica Ascendente e a Classificação Não Hierárquica por Partição, realizadas no software estatístico SPAD-N (Sistema Portátil de Análise de Dados - Programa 
Presta/Universidade Livre de Bruxelas) e no pacote computacional EPI INFO 6.2.

A escolha do método deve-se à natureza qualitativa das variáveis selecionadas e sua adequação para estudos onde se pretenda estabelecer a formação de grupos homogêneos a partir de múltiplas características.

A primeira análise destina-se à construção de uma tabela de casos e variáveis com valores quantitativos observados nos eixos fatoriais. Essa etapa é necessária, pois a tabela original é composta por atributos categóricos, não numéricos, que não podem ser submetidos à classificação.

A classificação hierárquica verifica o número de grupos no conjunto através das comparações entre os casos e sua proximidade ao centro de gravidade de cada grupo, garantindo a homogeneidade entre os semelhantes. Nesta etapa é possível identificar o número de grupos mais adequado para o conjunto de casos. Como passo final do agrupamento foi utilizado o Método de Classificação Não Hierárquica de Partição, que permitiu a alocação estável dos casos em quatro grupos, segundo sua proximidade ao centro de gravidade, bem como a amplitude da dispersão intergrupos. Trata-se de um procedimento em que os elementos são agrupados e reagrupados 3 a 4 vezes até atingirem uma estabilidade espacial do seu posicionamento, no sentido de garantir a máxima homogeneidade intra-grupo e heterogeneidade inter-grupo.

A análise dos 771 questionários foi para estabelecer a associação das variáveis com o fato de ter ou não ter LERs/DORTs e a análise das 437 respostas positivas para essas patologias foi de associação das variáveis entre si, indicando apenas quais as variáveis de identificação e perfil estavam associadas aos níveis de gravidade ou tempo das LERs/DORTs.

\section{Resultados e discussão}

Com relação aos questionários enviados, inicialmente, foi identificado o perfil dos cirurgiões-dentistas onde variáveis como sexo, idade, número de filhos, estado civil, tempo de graduação, especialidade etc., foram pesquisados.

Dos 771 questionários recebidos, $66,02 \%$ foram enviados por cirurgiõesdentistas do sexo masculino e os $33,98 \%$ restantes, enviados por profissionais do sexo feminino.

Observou-se uma concentração de profissionais entre 30 a 39 anos e entre 40 a 49 anos com $41,70 \%$ e $27,50 \%$, respectivamente, ou seja, nas faixas etárias de maior produtividade dos cirurgiões-dentistas. Zétola, em análise epidemiológica de 125 casos de LERs/DORTs em trabalhadores de indústria eletrônica, encontrou $84 \%$ das patologias nessa faixa etária, reforçando a característica crônica das patologias ${ }^{46}$.

Ao serem questionados quanto ao estado civil, a maioria dos entrevistados informou ser casada, 74,60\%. Esse valor equivalente a praticamente $3 / 4$ da amostra encontra resposta na concentração de profissionais entre 30 e 49 anos, ou seja, profissionais em uma faixa etária onde normalmente os indivíduos já estão com família constituída.

Quanto ao tempo de graduação em odontologia observou-se uma concentração de 46,40\% de profissionais com 10 a 19 anos de formados, ou seja, quase metade dos cirurgiões-dentistas está exposta, a princípio, a um período de tempo relativamente longo para adquirir algum tipo de LER/DORT.

Em relação ao tempo de efetivo exercício da profissão, os valores encontrados contribuem para demonstrar a consistência da amostra, pois o tempo de graduação e o tempo de efetivo exercício profissional apresentaram distribuição semelhante, ressaltando que $46,40 \%$ dos profissionais possuem entre 10 a 19 anos de graduação.

No que se refere aos profissionais que exerciam exclusivamente uma especialidade odontológica e se em caso afirmativo qual seria e o tempo de exercício na especialidade, $24 \%$ responderam que exerciam 
exclusivamente uma especialidade odontológica.

Entre os profissionais que responderam exercer exclusivamente uma especialidade odontológica observou-se que a grande maioria, $76,20 \%$, não respondeu qual especialidade, bem como $77,30 \%$ não informaram o tempo de exercício na especialidade. Assim, em virtude de grande parte dos entrevistados não ter respondido a estas perguntas, pouco se pôde conhecer sobre esses aspectos.

Ao serem inquiridos se apresentavam ou não alguma manifestação dolorosa nos membros superiores, cintura escapular ou pescoço, em virtude da repetição de um mesmo padrão de movimento no exercício da profissão, 437 (56,68\%) responderam que sim, e de 334 (43,32\%) foram obtidas respostas negativas. Esses valores são semelhantes aos encontrados por Santos Filho, com 58\% de cirurgiões-dentistas com queixa de dor músculo-esquelético em uma ou mais regiões do segmento superior do corpo ${ }^{33}$. Kosmann encontrou 81,51\% desses profissionais queixando-se de algum tipo de dor ou desconforto físico ${ }^{47}$.

Entre os profissionais que responderam apresentar algum sinal ou sintoma de LER/DORT, 59,5\% eram do sexo masculino e $40,5 \%$ do sexo feminino.

Embora os cirurgiões-dentistas do sexo masculino tenham apresentado valores absolutos superiores aos do sexo feminino, proporcionalmente foram encontra- dos mais casos de LERs/DORTs entre os profissionais do sexo feminino, o que está de acordo com Regis Filho e Lopes; Facci, Musse, Lech, Oliveira; Oliveira; Couto; Santos Filho; e Zétola ${ }^{11,20-22,33,46}$.

A associação entre as variáveis que descrevem as patologias, todas da terceira parte do protocolo, destinou-se a identificar as características do quadro de LERs/ DORTs, nos profissionais que referem impotência funcional.

A primeira análise foi para verificação de diferenças entre os indivíduos que responderam não apresentar e apresentar LERs/DORTs. Apenas as questões de identificação pessoal e perfil profissional foram respondidas pelos dois grupos. As freqüências simples e relativas de cada variável indicaram:

- presença de associação, teste Qui-quadrado, estatisticamente significante entre os dois sexos e presença de LERs/ DORTs. O sexo feminino apresenta mais patologias que o masculino. $\mathrm{O}$ valor do $\mathrm{c}^{2}$ foi 19,12 e p = 0,00001225, e - ausência de associação estatisticamente significante para as demais variáveis.

A segunda análise foi para identificação das características específicas daqueles que responderam apresentar LERs/ DORTs, onde o teste de Qui-quadrado indicou significância estatística para os cruzamentos apresentados no Quadro 1. Entretanto, por estarem sendo analisados

Quadro 1 - Identificação das Características Específicas dos Indivíduos que Apresentam Sinais e Sintomas de LERs/DORTs.

Chart 1 - Specific Characteristics of Individuals with Signs and Symptoms of CTD/WRMD.

\begin{tabular}{lccc}
\hline Cruzamento & GL & Qui-quadrado & Valor de p \\
\hline Sexo x Intensidade da Dor & 2 & 9,679 & 0,0079 \\
Sexo x Duração da Dor & 2 & 14,446 & 0,0007 \\
Sexo x Dor Provocada & 3 & 14,460 & 0,0023 \\
Idade x Tempo que tem Dor & 4 & 49,000 & $<0,0001$ \\
Idade x Sinais & 2 & 8,433 & 0,0037 \\
Idade x Tratamento & 2 & 10,055 & 0,0015 \\
Tempo de Exercício Profissional x Tempo que tem Dor & 8 & 76,244 & $<0,0001$ \\
Tempo de Exercício Profissional x Sinais & 2 & 17,975 & 0,0001 \\
Tempo de Exercício x Tratamento & 2 & 14,489 & 0,0007 \\
\hline
\end{tabular}


apenas os casos com LERs/DORTs, as inferências de causalidade e mesmo de prevalência, devem ser feitas dentro de critérios mais rígidos.

Embora não exista associação estatisticamente significante para a variável local inicial da dor, é interessante analisar esta variável que pode ser um primeiro indicativo da região da ocorrência da LER/ DORT. A maior ocorrência foi para a região de ombro/braço, 39,40\%, seguida da região do punho/mão, 18,30\%, e pescoço com $17,20 \%$. Zétola ${ }^{46}$ encontrou maior ocorrência para tendinite de punho, 45,9\%, seguida da região do ombro, 18,5\%, o que está parcialmente de acordo com os resultados do presente trabalho.

Números semelhantes foram observados no estudo realizado por Regis Filho e Lopes, onde os ombros e punhos, ambos em $44,44 \%$ cada um, foram os locais em que os homens relatavam maior sintomatologia dolorosa. De acordo com os autores, $57,89 \%$ e $36,84 \%$ das mulheres apresentavam dor nos punhos/dedos e ombros, respectivamente ${ }^{11}$.

Os valores encontrados por Santos Filho $^{33}$, em que $22 \%, 21 \%, 20 \%$ e $17 \%$ apresentavam dor no membro superior, coluna, pescoço e ombro, respectivamente, estão parcialmente de acordo com o presente trabalho. A distribuição percentual dos locais das patologias pode ser observada no Gráfico I.

Os cruzamentos para a variável sexo in- dicaram diferença apenas para intensidade da dor, duração da dor e dor provocada. $\mathrm{O}$ teste indicou, também, que o sexo feminino tem menos dor de intensidade fraca que o sexo masculino. Entretanto, a dor, para o sexo feminino, está distribuída entre intensidades média e forte. Para a duração da dor, as mulheres apresentaram menos dor do tipo fugaz que os homens, tendo maior quantidade de dor do tipo intermitente e constante. A dor provocada foi investigada quanto ao movimento e à palpação. O sexo feminino apresentou menos dor provocada por movimento e mais dor provocada por palpação do que o esperado.

Os cruzamentos para a variável idade indicaram diferença para tempo que apresenta dor, sinais e tratamentos. Na análise de tempo que apresenta dor, a variável idade foi agrupada em duas faixas, até 40 e mais de 40 anos, para permitir cruzamentos com número menor de graus de liberdade. Observou-se que profissionais com mais de 40 anos têm dor há mais tempo que os profissionais com menos de 40 anos. Para sinais apresentados, o teste indicou que cirurgiões-dentistas com mais de 45 anos apresentavam mais sinais de que os profissionais com menos idade. Essa característica é ainda mais definida para indivíduos com mais de 50 anos. A mesma tendência foi verificada para tratamentos realizados, mas já aos 40 anos é percebido um maior número de tratamentos que o esperado.

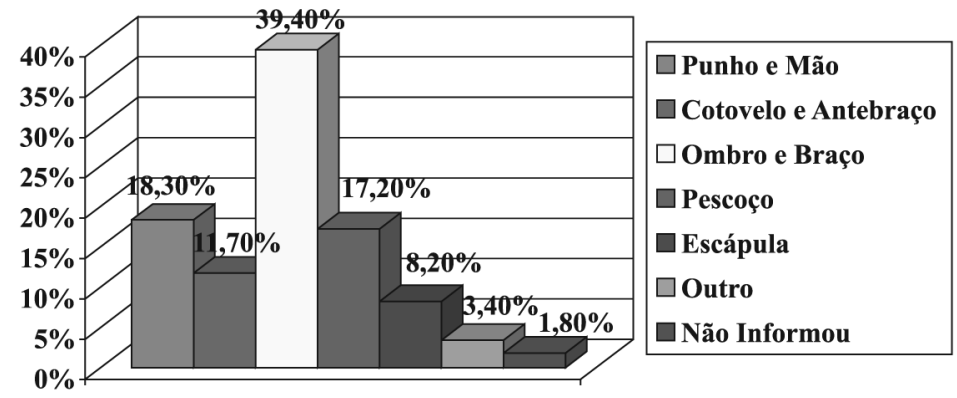

Gráfico I - Distribuição dos Casos de Lesão por Esforço Repetitivo em Cirurgiões- Dentistas, segundo a Localização Inicial da Dor.

Graph I - Distribution of Cases of Cumulative Trauma Disorder in Dentists, according to initial location of Pain. 
Quanto ao tempo de exercício da profissão, foram observadas as mesmas associações que para a idade, o que era esperado dada a alta associação entre as duas variáveis. Para essa análise, o tempo de exercício profissional foi classificado em três categorias: até 10 anos, maior que 10 e até 20 anos e maior que 20 anos. O verificado para tempo que apresenta dor foi que os profissionais que têm até 10 anos de exercício da profissão têm maior número de casos com dor nas faixas de 1, 2 ou 3 anos; aqueles com até 20 anos de exercício apresentaram maior número de casos nas faixas com 3 a 5 anos ou mais; e o grupo com mais de 20 anos de exercício teve maior número de casos com mais de 5 anos de dor. Para os sinais, o grupo com até 10 anos de exercício apresentou menor número de casos com sinais e o grupo com mais de 20 anos de exercício apresentou maior número de casos com sinais que o esperado. Comportamento idêntico foi observado para tratamento.

A análise para verificação da associação entre as variáveis para identificar as características do quadro de LERs/DORTs nos profissionais que referem impotência funcional indicou as associações apresentadas na Quadro 2.

Os resultados dos testes aplicados indicaram que a impotência funcional está associada com dor no início da atividade, de intensidade forte e intermitente, existindo dor provocada pelo movimento repetitivo e pela palpação. Por outro lado, existem poucos casos com fatores que melhoram a dor. A impotência funcional também está associada à presença de sinais e entre os sinais, a perda de controle dos movimentos é o que está mais associado.

Existe um maior número de tratamentos realizados nos casos que referem impotência funcional, e entre os profissionais que se submetem a tratamento a fisioterapia aparece em número maior que o esperado para os casos com impotência funcional. Isso pode indicar que a fisioterapia é um tratamento muito procurado, entretanto não resolve o problema, pois as condições do ambiente de trabalho permanecem inalteradas.

A análise fatorial de correspondência múltipla, seguida de classificação hierárquica e de partição, identificou quatro grupos distintos de profissionais com LERs/ DORTs em relação aos sintomas, a saber: baixa, média e alta severidade com tratamento fisioterápico, e alta severidade com tratamento cirúrgico. Para essa análise, foram desconsideradas as variáveis que apresentaram mais de $10 \%$ de respostas "não informado". As variáveis eliminadas foram: membros afetados, tempo que o indivíduo tem dor, início da dor e agravamento da dor. Além dessas, as variáveis idade e tempo de graduação foram suprimidas pela alta correlação com o tempo de

Quadro 2 - Associação entre as Variáveis para Identificar as Características do Quadro de LERs/ DORTs, em Relação a Impotência Funcional.

Chart 2 - Association among Variables to identify the Characteristics of CTD/WRMD in Relation to Functional Incapacity.

\begin{tabular}{lccc}
\hline Impotência Funcional & GL & Qui-quadrado & Valor de p \\
\hline Início da Dor & 2 & 10,773 & 0,0046 \\
Intensidade da Dor & 2 & 20,659 & $<0,0001$ \\
Duração da Dor & 2 & 8,352 & 0,0154 \\
Dor Provocada & 3 & 30,246 & $<0,0001$ \\
Fatores que Alteram a Dor & 3 & 13,754 & 0,0033 \\
Presença de Sinais & 1 & 74,937 & $<0,0001$ \\
Perda de Controle dos Movimentos & 1 & 25,516 & $<0,0001$ \\
Ter Feito Tratamento & 1 & 13,291 & 0,0003 \\
Tipos de Tratamentos & 5 & 13,514 & 0,0190 \\
\hline
\end{tabular}


Quadro 3 - Distribuição dos Profissionais com Sintomatologia de LERs/DORTs.

Chart 3 - Distribution of Professionals with Symptoms of CTD/WRMD.

\begin{tabular}{|c|c|c|}
\hline GRUPOS & No & CARACTERÍSTICAS \\
\hline 1 & 105 & $\begin{array}{l}64 \% \text { dos profissionais têm dor fraca, o que corresponde a } 74 \% \text { de todos os casos de dor } \\
\text { fraca analisados; } 66 \% \text { dos casos têm dor fugaz; } 77 \% \text { não apresentam qualquer sinal; } 56 \% \\
\text { não fizeram tratamento algum; } 86 \% \text { não têm impotência funcional; } 79 \% \text { são do sexo } \\
\text { masculino; tempo de exercício profissional mais concentrado na faixa de } 5 \text { a } 10 \text { anos; } \\
\text { outras variáveis apresentaram menor contribuição para formação do grupo. }\end{array}$ \\
\hline 2 & 149 & $\begin{array}{l}89 \% \text { dos profissionais têm dor de média intensidade; } 91 \% \text { dos casos não têm impotência } \\
\text { funcional; concentra } 84 \% \text { dos casos que apresentam dor à palpação; } 66 \% \text { dos casos } \\
\text { apresentam dor constante; } 60 \% \text { são mulheres, correspondendo a } 52 \% \text { do total; } 62 \% \text { dos } \\
\text { casos apresentam alteração de sensibilidade e } 50 \% \text { dos casos deste grupo não apresentam } \\
\text { sinais; sendo o tempo de exercício profissional distribuído nas faixas de } 5 \text { a } 20 \text { anos. }\end{array}$ \\
\hline 3 & 152 & $\begin{array}{l}\text { A impotência funcional é a variável de maior contribuição para formação do grupo, com } \\
62 \% \text { no grupo, o que corresponde a } 87 \% \text { do total de casos de impotência funcional; } 63 \% \\
\text { dos profissionais fizeram tratamento fisioterápico; os sinais são importantes, com } 89 \% \text { do } \\
\text { total de casos de perda de controle dos movimentos, } 68 \% \text { dos casos de limitação de } \\
\text { movimento e } 57 \% \text { dos casos de edema; concentra } 73 \% \text { dos casos de dor forte; } 62 \% \text { dos } \\
\text { casos apresentam dor ao movimento e à palpação; tempo de exercício concentrado nas } \\
\text { faixas de } 20 \text { anos ou mais. }\end{array}$ \\
\hline 4 & 20 & $\begin{array}{l}\text { O tipo de tratamento definiu o grupo, com } 100 \% \text { dos casos de cirurgia; porém outras } \\
\text { varáveis são relevantes; } 65 \% \text { destes casos são do sexo masculino, } 75 \% \text { com mais de } 40 \text { anos } \\
\text { de idade e mais de } 15 \text { anos de exercício profissional. }\end{array}$ \\
\hline
\end{tabular}

Quadro 4 - Distribuição Percentual dos Casos de LERs/DORTs, em Relação a Severidade. Chart 4 - Case Distribution of CTD/WRMD in Relation to Severity.

\begin{tabular}{|c|c|c|}
\hline Severidade & Valores Absolutos & $\%$ \\
\hline Baixa & 105 & 24,65 \\
\hline Média & 149 & 34,98 \\
\hline Alta com Fisioterapia & 152 & 35,68 \\
\hline Alta com Cirurgia & 20 & 4,69 \\
\hline Total & 426 & 100,00 \\
\hline
\end{tabular}

exercício da profissão $\left(\mathrm{r}_{\mathrm{s}}=0,93-\mathrm{p}<0,0001\right)$.

Foram eliminadas, também, as variáveis estado civil e tempo de especialidade, em virtude do baixo nível de variabilidade, não contribuindo para a análise. Por outro lado, foram eliminados 11 casos que apresentaram respostas "não informado" para todas as variáveis características de dor. As principais características de cada grupo estão descritas no Quadro 3.

A distribuição mais ou menos harmônica dos casos, em relação a severidade baixa, média e alta, correspondentes aos graus I, II e III da classificação dos estágios das LERs/DORTs, indica que as tarefas executadas na prática odontológica favorecem a evolução das patologias. O quarto grupo, aqueles que se submetem a tratamento cirúrgico, equivale ao grau IV.

A partir dos números observados no Quadro 3 pode-se obter a distribuição percentual em relação à severidade dos sintomas, conforme apresentado no Quadro 4.

\section{Conclusões}

No presente trabalho, ao se analisar a população estudada a partir do estudo 
epidemiológico transversal, observa-se uma forte predominância do sexo masculino, e uma concentração acentuada de profissionais na faixa etária entre 30 e 49 anos, $69,20 \%$. A grande maioria dos entrevistados informa ser casado e ter entre 10 a 19 anos de graduação em odontologia, $46,40 \%$, sendo que um percentual semelhante de profissionais, $45,50 \%$, exerce efetivamente a profissão nesse mesmo espaço de tempo.

A análise estatística indica presença de associação estatisticamente significante entre os dois sexos para presença de LERs/ DORTs, sendo que o sexo feminino apresenta mais patologias que o masculino. Por outro lado, a análise estatística indica ausência de associação estatisticamente significante para as demais variáveis pesquisadas. Ombro/braço (39,40\%), punho/mão (18,30\%) e pescoço $(17,20 \%)$, são as regiões mais afetadas por LERs/DORTs.

A partir da análise estatística também se pode agrupar a distribuição dos casos de LERs/DORTs em quatro grandes grupos, com características bastante evidentes, e com distribuição mais ou menos harmônica dos casos em relação a severidade:

- primeiro grupo: de baixa severidade, caracterizado por sexo masculino, ausência de sinais, tratamento e impedimento funcional, apresentando dor fraca e de duração fugaz;

- segundo grupo: de média severidade, caracterizado por sexo feminino, sem impedimento funcional, apresentando dor de intensidade média, duração constante e durante a palpação, e com presença de sensibilidade;

- terceiro grupo: de alta severidade, tem impedimento funcional, apresentando dor forte e intermitente, presença de diversos sinais como limitação perda de controledos movimentos e edema, tratamento fisioterápico e longo tempo de exercício profissional; e

- quarto grupo: de alta severidade, com predominância do sexo masculino, apresenta as mesmas características do terceiro grupo, sendo que o tratamen- to cirúrgico o diferencia do grupo anterior.

Os quatro grupos mostram uma característica da profissão, onde os cirurgiõesdentistas permanecem trabalhando, mesmo apresentando evolução da patologia para os graus de alta severidade, porém para que isso possa ocorrer submetem-se a fisioterapia. Entretanto, como o tratamento não leva à resolução do problema, em virtude das características próprias da profissão, como o exercício da clínica privada, uma parcela de profissionais recorre ao tratamento cirúrgico para poder continuar exercendo suas atividades.

A metodologia empregada no presente estudo mostrou-se confiável, exeqüível e adequada para testar a hipótese de trabalho, pois permitiu a aquisição de um significativo volume de informações, possibilitando conhecer os fatores de risco do trabalho em odontologia.

Os resultados obtidos confirmam a hipótese de trabalho e permitem afirmar que o cirurgião-dentista pertence a um grupo profissional exposto a risco considerável de adquirir algum tipo de LER/DORT, desde que certos fatores inerentes às tarefas profissionais, aí consideradas força excessiva, posturas incorretas, alta repetitividade de um mesmo padrão de movimento e compressão mecânica dos tecidos, aliadas às características individuais, estejam presentes.

Os resultados obtidos indicam, ainda, que a maioria dos cirurgiões-dentistas em virtude da utilização de instrumentos que não obedecem a requisitos ergonômicos e da realização de tarefas inadequadamente prescritas, entre outros fatores, estão sendo submetidos a condições adversas de trabalho, onde dor e desconforto estão presentes.

Fazem-se necessários estudos que possibilitem o redesenho das tarefas para permitir a redução de tensões biomecânicas sobre os membros superiores e regiões adjacentes e que incentivem a criação de instrumentos obedecendo a requisitos 
ergonômicos. O projeto de novos instrumentos deve ter por finalidade, entre outras, evitar altas forças de contato e posicionamento estático, posturas extremas ou inadequadas das articulações, movimentos repetitivos dos dedos e vibrações deletérias. O instrumento não deve ser simplesmente efetivo para a realização de determinada tarefa, mas também precisa ser julgado adequado pelo usuário em termos de balanço, peso, forma, vantagem biomecânica, sensibilidade ao contato e no modo de utilização, minimizando o desenvolvimento do processo de fadiga e da tensão acumulada ao longo do dia de trabalho.

Por outro lado, a comprovação da associação de LERs/DORTs com as tarefas exercidas por um grupo profissional e que até então não era praticamente citado na literatura, é fator relevante não só na prevenção das doenças de caráter ocupacional, mas também para os aspectos legais, pois, atualmente, os planos de saúde ainda não oferecem cobertura para essas patologias em cirurgiões-dentistas.

Finalizando, sugere-se que mais e intensas pesquisas devam ser realizadas com a finalidade de aprofundar o debate, principalmente aquelas abordando fatores psicossociais, culturais, de organização temporal das tarefas, relativas à antropometria para possibilitar a confecção de instrumentos e equipamentos mais adequados às dimensões corporais dos profissionais brasileiros e por fim utilizando a dinamometria, para mensurar a força e a pressão exercidas sobre instrumentos de uso freqüente na prática odontológica.

\section{Referências}

1. Huberman L. História das riquezas do homem. $21^{\mathrm{a}} \mathrm{ed}$. rev. Rio de Janeiro: Editora Guanabara; 1986.

2. Taylor WF. Princípios de administração científica. São Paulo: Atlas; 1987.

3. Fayol H. Administração industrial e geral. São Paulo: Atlas; 1989.

4. Juran JM. Controle da qualidade: conceitos, políticas e filosofia da qualidade. São Paulo: Makron Books; 1992.

5. Deming WE. A qualidade: revolução da administração. São Paulo: Marques Saraiva; 1997. 205p.

6. Drucker PF. As novas realidades: no governo e na política, na economia e nas empresas, na sociedade e na visão do mundo. $3^{\text {a }}$ ed. São Paulo: Pioneira; 1993.

7. Ishikawa K. Controle de qualidade total a maneira japonesa. São Paulo: Campus/ 1998.

8. McGregor D. O lado humano da empresa. São Paulo: M. Fontes; 1992.

9. Etzioni A. Organizações modernas. São Paulo: Pioneira; 1989.

10. Ferguson DA. "RSI": putting the epidemic to rest. Med J Aust 1987; 147; 213-4

11. Regis Filho GI, Lopes MC. Aspectos epidemiológicos e ergonômicos de lesões por esforço repetitivo em cirurgiões-dentistas. Rev APCD 1997; 51(5): 469-75.
12. McKeag. Overuse injuries. Prim Care 1991; 18(4): 851-65.

13. Rocha LEM, Paes EM, Sobania LC. Lesões por esforço de repetição: análise em 166 digitadores de um centro de computação de dados. Rev Bras Ortop 1986; 21(4); 115-9.

14. Maciel RH. Considerações gerais sobre o trabalho de digitação. Rev Bras Saúde Ocup 1985; 13(50); 37-40.

15. Feuerstein M, Fitzgerald T. Biomechanical factors affecting upper extremity cumulative trauma disorders in sign language interpreters. J Occup Med 1992; 34(3); 25764 .

16. Matte TD, Baker, EL, Honchar PA. The selection and definition of target work-related conditions for surveillance under Sensor. Am J Public Health 1989; 79(S); 21-5.

17. Erdil M, Dicerson OB, Glackin E. Cumulative trauma disorders of the upper extremity. In: Zenz C. Occupational Medicine. $3^{\mathrm{a}}$ ed. St. Louis: Mosby; 1994. p.48-64.

18. Hadler NM. Cumulative trauma disorders: an iatrogenic concept. JOccup Med 1990; 32(1); 38-41.

19. Malloryy M, Bradford . An invisible workplace hazard gets harder to ignore. Business Week 1989; 30; 92-3.

20. Facci RC, Musse CA, Lech O, Oliveira R. A síndrome do excesso de uso. Proteção 1989; 1(6): 41-4.

21. Oliveira CR. Lesão por esforço repetitivo (L.E.R.). Rev Bras Saúde Ocup 1991; 19(73): 59-85. 
22. Couto H. de A. Doenças profissionais: guia prático de tenossinovites e outras doenças de origem ocupacional. Belo Horizonte: Asta Médica; 1994.

23. Barreira TH de C. Abordagem ergonômica na prevenção da ler. Rev Bras Saúde Ocup 1994; 22(84): 51-60.

24. Codo W, Almeida MCCG de. L.E.R: diagnóstico, tratamento e prevenção. uma abordagem interdisciplinar. Petrópolis: Vozes; 1997.

25. Assunção AA. Sistema músculo-esquelético: lesões por esforços repetitivos (LER). In: Mendes R. Patologia do Trabalho. Rio de Janeiro: Atheneu; 1997. p.173-212.

26. INSS. Diário Oficial da União. Brasília, 15/08/88, n. 158, p. 26-34.

27. Cunha CEG, Queiroz OS, Hatem TP, Guimarães VYM. L.E.R. lesões por esforços repetitivos: revisão. Rev Bras Saúde Ocup 1992; 20(76): 47-59.

28. Andersen J, Gaardboe O. Musculoskeletal disorders of the neck and upper limb among sewing machine operators: a clinical investigation. Am J Ind Med 1993; 24(6): 689-700.

29. Eccles JD, Powell M. The health of dentists. Br Dent J 1967; 123(12): 379-87.

30. Lawrence JS. Rheumatic diseases. International Labour office Encyclopaedia of Occupational Health and Safety 1972; 2(1): 1233-4.

31. Saquy PC, Pécora JD. Orientação profissional em odontologia. São Paulo: Santos; 1996.

32. Nogueira DP. Riscos ocupacionais de dentistas e sua prevenção. Rev Bras Saúde Ocup 1983; 11(41); 14-6.

33. Santos Filho SB. Prevalência de dor osteomuscular de membros superiores em cirurgiões-dentistas do serviço público de Belo Horizonte: contribuição no debate sobre os distúrbios osteomusculares relacionados ao trabalho (DORT) [dissertação de mestrado]. Belo horizonte: Faculdade de Saúde Públic da UFMG; 1998.

34. Bauer ME. Carpal tunnel syndrome. an occupational risk to the hygienist. Dent Hyg 1985; 59(5): 218-21.

35. Holtzhausen T. Carpal tunnel syndrome. A "new" occupational hazard for the oral hygienist. Occup Hazards 1986; 76(6): 33-4.

36. Lopes A, Villanacci Neto R. A síndrome do túnel carpal: um risco profissional para o cirurgião-dentista. Rev $A P C D$ 1994; 48(6); 1545-52.
37. Cândido L, Bittencourt MZ, Regis Filho GI. Lesões por Esforço Repetitivo em Cirurgiões-Dentistas: Um Estudo de Caso - Cisto Sinovial de Punho. J Br Cli Odont Int Curitiba 2003; 7(42); 463-6.

38. Martins CA, Fadel MAV, Regis Filho GI. Lesões por Esforço Repetitivo em Cirurgiões-Dentistas: Um Estudo de Caso - Síndrome do Túnel Carpal. J Br Cli Odont Int Curitiba 2003; 7(41): 363-7.

39. Fadel MAV, Petrobon L, Patiño JSR, Regis Filho GI. Lesões Por Esfoço Repetitivo em Cirurgiões-Dentistas: Um Estudo de Caso - Dedo em Gatilho. Rev APCD 2006; 60(S); 189.

40. Barnhart S, Demers PA, Miller M, Longstreth WT Jr; Rosenstock L. Carpal syndrome among ski manufacturing workers. Scand J Work Environ Health 1991; 17(1): 46-53.

41. Jetzer TC. Use of vibration testing in the early evaluation of workers with carpal tunnel syndrome. J Occup Environ Med 1991; 33(2): 117-20.

42. Regis Filho GI, Fadel MAV, Pietrobon L, Zmijvski TRL, Klug FK. Exposição Ocupacional do Cirurgião-dentista à Vibração Mecânica Transmitida Através das Mãos - Um Estudo de Caso. Rev APCD 2006; 60(S); 188.

43. Pece CAZ. Concepção ergonômica, desenvolvimento e otimização de um fórceps odontológico: proposta de nova sistemática exodôntica [dissertação de mestrado]. Faculdade de Engenharia Aeronáutica e Mecânica, ITA. 1995.

44. Lech O, Hoefel MG. Protocolo de investigação das lesões por esforços repetitivos (L.E.R.). Belo Horizonte: Rhodia Farma; 1992.

45. EBCT. Empresa Brasileira de Correios e Telegráficos. Diretoria Regional de Santa Catarina. Manual Carta/ Cartão-Resposta. Florianópolis, 1998.

46. Zétola PR. Análise epidemiológica de 125 casos de lesões por esforços repetitivos - L.E.R. em trabalhadores de indústria eletrônica [dissertação de mestrado]. Faculdade de Engenharia da Produção da UFSC; 2000.

47. Kosmann C. Dor e desconforto no trabalho do dentistacontribuições da ergonomia [dissertação de mestrado]. Faculdade de Engenharia da Produção da UFSC; 2000.

recebido em: 09/10/05 versão final reapresentada em: 10/05/06 aprovado em: 13/05/06 